\title{
THE CLOSING LEMMA AND STRUCTURAL STABILITY
}

\author{
BY CHARLES C. PUGH ${ }^{1}$ \\ Communicated by L. Markus, March 30, 1964
}

Introduction. Consider a differentiable $n$-manifold $M$. Let $x=\mathscr{X}(M)$ be the space of all $C^{1}$ tangent vector fields on $M$ under a $C^{1}$ topology [1]. Each $X \in X$ induces a $C^{1}$-flow on $M$ called the $X$-flow. Let $d$ be a metric on $M$ and let $\epsilon$ be positive. Two flows are homeomorphic if there is a homeomorphism $h$ of $M$ onto itself taking the trajectories of one flow onto those of the other; the two flows are $\epsilon$-homeomorphic if $h$ can be chosen so that $d(h(p), p)<\epsilon$ for all $p \in M$. X is said to be structurally stable if, given $\epsilon>0$, there then exists a neighborhood $\mathcal{u}$ of $X$ in $X$ such that for each $Y \in \mathcal{u}$ the $Y$-flow is $\epsilon$-homeomorphic to the $X$-flow. Let us say that $X$ is crudely structurally stable if we drop the $\epsilon$ condition: $X$ is crudely structurally stable if there exists a neighborhood $\mathcal{U}$ of $X$ in $\mathscr{X}$ such that $Y \in \mathcal{U}$ implies that the $Y$-flow is homeomorphic to the $X$-flow. Let $\Sigma$ denote those $X$ in $X$ which are structurally stable and let $\Sigma_{c}$ denote those $X$ in $X$ which are crudely structurally stable, obviously $\Sigma \subset \Sigma_{c}$. The problem of structural stability theory is to characterize $\Sigma$ and $\Sigma_{c}$ and to study the topological relation of $\Sigma$ and $\Sigma_{c}$ to $x$.

The most comprehensive results in structural stability theory are due to M. Peixoto [2], [3], [4] who has shown, when $M$ is a compact 2 -manifold, that $\Sigma=\Sigma_{c}, \bar{\Sigma}=\mathscr{X}$, and that the fields in $\Sigma$ are characterized completely as the fields with "generic" induced flows.

Related to the problem of structural stability is the following conjecture:

Closing Lemma. If the $X$-flow has a nontrivial recurrent trajectory through some $p \in M$ and if $u$ is any neighborhood of $X$ in $x$ then there exists $Y \in \mathcal{U}$ such that the $Y$-flow has a closed orbit through $p$.

(Recall that a trajectory is nontrivially recurrent if it is contained in its $\alpha$ - or in its $\omega$-limit set without being a closed orbit or a stationary point.)

Results concerning the Closing Lemma. M. Peixoto [4] has proved the Closing Lemma in the case that $M$ is the 2-torus and $X$ has no

${ }^{1}$ The author holds a fellowship from the United States Steel Foundation at The Johns Hopkins University. This research was also supported in part by the Air Force Office of Scientific Research. I am glad to thank Dr. M. M. Peixoto for his invaluable guidance, and Dr. P. Hartman for helpful criticism. Thanks are also due to M. L. Peixoto, I. Kupka, G. Sottomayor, and A. Barreto for useful conversa tions at IMPA. 
singularities. We prove the following two forms of the Closing Lemma. (Our proofs, however, are invalid for a $C^{r}$ topology on $\mathscr{X}^{r}, r>1$.)

Theorem 1. Let $M$ be any differentiable 2-manifold and let $X \in X$ have a nontrivial recurrent trajectory through $p \in M$. Let $U$ be an arbitrarily small coordinate neighborhood of $p$ in $M$ and let $\epsilon>0$ be given. Then there exists $\Delta \in X$ such that

(a) $\Delta$ vanishes on $M-U$.

(b) The $C^{1}$ size of $\Delta$ respecting the coordinates of $U$ is less than $\epsilon$.

(c) $Y=X+\Delta$ has a closed orbit through $p$.

Theorem 2. Let $M$ be a compact n-manifold and let a Riemannian metric be put on $M$ so that the norm of each linear transformation $L: T_{x}(M) \rightarrow T_{y}(M)$ is defined. Suppose that $X \in X$ induces a flow $\phi$ which has a nontrivial recurrent trajectory through $p \in M$. Define $J(t, x)$ : $T_{x}(M) \rightarrow T_{\phi(t, x)}(M)$ to be the jacobian isomorphism of tangent spaces induced by $x \rightarrow \phi(t, x)$. Suppose that $\epsilon>0$ is given and that

$$
\lim _{t \rightarrow \infty} \frac{1}{t}\left\|J^{-1}(t, p)\right\|=0 .
$$

Then there exists $\Delta \in X$ such that the $C^{1}$ size of $\Delta$ is less than $\epsilon$ and $Y=X+\Delta$ has a closed orbit through $p$.

Where $M$ is compact, all Riemannian metrics are equivalent and so Theorem 2 does not depend on the choice of Riemannian metric.

Definition. Let $X$ be in $x(M)$ for a differentiable $n$-manifold $M$. $A$ flow-box for $X$ at $p \in M$ is a coordinate neighborhood $U$ of $p$ in $M$ such that in terms of the coordinates $\left(u^{1}, \cdots, u^{n}\right)$ of $U, u^{i}(p)=0$ for $i=1,2, \cdots, n$ and

$$
X_{u}=\left(\frac{\partial}{\partial u^{1}}\right)_{u} \quad \text { for all } u \text { in } U .
$$

If $X_{p} \neq 0$, then it is well known that a flow-box for $X$ at $p$ exists.

The following lemma is the principal tool used to prove Theorems 1 and 2 .

Lemma. Let $\epsilon>0$ and $0<\delta<1$ be given. Let $M$ be a differentiable nmanifold and let $X \in X$ induce the flow $\phi$. Suppose that $X$ does not vanish at $p^{*} \in M$ and let $U$ be a flow-box for $X$ at $p^{*}$. Let

$$
\Pi=\left\{\left(0, u^{2}, u^{3}, \cdots, u^{n}\right) \in U\right\} .
$$

Suppose that $P$ is a subset of $\Pi$ such that arbitrarily near $p^{*}$ there are distinct points of $P$ lying on a common $\phi$-trajectory (e.g., let $P=\mu \cap \Pi$ 
and let $p^{*} \in \bar{\mu} \cap \Pi$ where $\mu$ is a nontrivial recurrent $\phi$-trajectory). Then there exist points $p$ and $q$ of $P$ such that

$$
\begin{aligned}
\left|p-p^{*}\right| & <\epsilon, \\
\left|q-p^{*}\right| & <\epsilon, \\
\phi\left(t^{*}, p\right) & =q \text { for some } t^{*}>0,
\end{aligned}
$$

and

$$
\begin{aligned}
& \text { If } r=\phi\left(t^{\prime}, p\right) \in P \text { for some } t^{\prime}, 0<t^{\prime}<t^{*}, \\
& \text { then }|p-r|>\delta|p-q| \text { and }|q-r|>\delta|p-q|,
\end{aligned}
$$

where $|x-y|$ denotes the distance between $x$ and $y$ respecting the coordinates of $U$.

The proof of this lemma is easy. Just take a $p_{0}$ and $q_{0}$ in $P$ obeying (a) where $\epsilon$ has been replaced by the smaller constant $\frac{1}{2}(1-\delta)$ $\cdot \epsilon$ and where $t^{*}$ is called $t_{0}$. If (b) fails to be true for some $r=\phi\left(t^{\prime}, p_{0}\right)$, then suppose that $\left|q_{0}-r\right| \leqq \delta\left|p_{0}-q_{0}\right|$. Replace $q_{0}$ by $r$ and regard the pair $\left(p_{0}, r\right)$ instead of the pair $\left(p_{0}, q_{0}\right)$. Call $\left(p_{0}, r\right)=\left(p_{1}, q_{1}\right)$. Proceed similarly if $\left|q_{0}-r\right|>\delta\left|p_{0}-q_{0}\right|$ but $\left|p_{0}-r\right| \leqq \delta\left|p_{0}-q_{0}\right|$ to get $\left(p_{1}, q_{1}\right)=\left(r, q_{0}\right)$. Proceed with $\left(p_{1}, q_{1}\right)$ as was done with $\left(p_{0}, q_{0}\right)$, getting, thereby, a sequence $\left(p_{k}, q_{k}\right) k=1,2, \cdots$. The process ends at a finite step $\left(p_{m}, q_{m}\right)$ because $\phi(t, p)$ crosses $\Pi$ at most a finite number of times for $0 \leqq t \leqq t_{0}$. The pair $\left(p_{m}, q_{m}\right)$ satisfies (b) by construction, It also satisfies (a) because

$$
\begin{aligned}
\left|p^{*}-p_{m}\right| & \leqq \sum_{i=1}^{m} \max \left(\left|p_{i}-p_{i-1}\right|,\left|q_{i}-q_{i-1}\right|\right)+\left|p_{0}-p^{*}\right| \\
& \leqq \sum_{i=1}^{m} \delta^{i}\left|p_{0}-q_{0}\right|+\left|p_{0}-p^{*}\right| \\
& <\left|p_{0}-q_{0}\right| \cdot \frac{1}{1-\delta}+\left|p_{0}-p^{*}\right| \\
& <\frac{\epsilon \cdot(1-\delta)}{2 \cdot(1-\delta)}+\frac{\epsilon(1-\delta)}{2}<\epsilon .
\end{aligned}
$$

Similarly $\left|p^{*}-q_{m}\right|<\epsilon$.

As a consequence of Theorem 1, M. Peixoto's paper [4] can be shortened considerably. The methods used to prove Theorem 1 can also be used to solve the following problem.

Suppose that $M=S^{2}, X \in \mathscr{C}\left(S^{2}\right)$, and that the $X$-flow has a closed orbit $\gamma$ which is isolated but unstable. Suppose there are $n$ generic saddle points $p_{1}, p_{2}, \cdots, p_{n}$ outside $\gamma$ and $n$ more generic saddle points $q_{1}, q_{2}, \cdots, q_{n}$ inside $\gamma$ such that one separatrix from each $p_{i}$ 
has $\gamma$ as an $\omega$-limit and one separatrix from each $q_{i}$ has $\gamma$ as an $\alpha$ limit point. The problem is to find an arbitrarily $C^{1}$ small $\Delta \in X$ such that for $Y=X+\Delta$, the $Y$-flow "joins the $p_{i}$ 's to the $q_{j}$ 's." That is, each $p_{i}$ should have a $Y$-separatrix $\sigma_{i}$ which is also a $Y$-separatrix of some $q_{j}$. When $\Delta$ is sufficiently $C^{1}$ small, it is easily seen that the same $q_{j}$ cannot be joined to two different $p_{i}$ 's. M. Peixoto [4] has solved this problem for $n=1$. The problem for $n \geqq 2$ is related to an investigation of "higher order structural stability" at present being completed by G. Sottomayor. Sottomayor wishes $\Delta$ to be $C^{5}$ small, but-as in the Closing Lemma itself-our methods only produce perturbations which are $C^{1}$ small.

I hope that Theorem 2 will yield as a corollary that distal minimal nontrivial recurrent flows on compact differentiable manifolds may be closed by arbitrarily $C^{1}$ small perturbations $\Delta$. It would suffice to prove that for some $p \in M,\left\|J^{-1}(t, p)\right\|$ is bounded as $t \rightarrow \infty$ where $J$ is the jacobian isomorphism induced as in Theorem 2. Roughly, the reason this should be true is that $\left\|J^{-1}\right\|$ is a measure of how fast the flow contracts and distal flows don't contract too much.

Finally, we inspect two examples related to the theory of structural stability for noncompact 2-manifolds. First we show that for $M=R^{2}$, $\Sigma_{c} \neq \Sigma$. Second, following M. L. Peixoto, we see that there exists a nonvanishing $X \in \mathfrak{X}\left(R^{2}\right)$ which is not in $\Sigma_{c}$. This shows that it will probably be quite difficult to characterize the elements of $\Sigma$ and $\Sigma_{c}$ for noncompact 2-manifolds.

In a sense, this is unfortunate because Theorem 1 holds for noncompact differentiable 2-manifolds and one might hope to use it to try to generalize M. Peixoto's characterization theorem [4] to the noncompact case. In particular one would hope to show that $X \in \Sigma_{c}$ if the $X$-flow has a nontrivial recurrent trajectory. I can prove this if $M$ has finite genus but if $M$ has infinite genus, I can prove it only by using the following

Conjecture. Suppose that $M$ is a differentiable 2-manifold and that $X \in \Sigma_{c}(M)$. Let $\Gamma$ be the union of all the closed orbits of the $X$-flow. Then $\Gamma$ is closed in $M$.

\section{REFERENCES}

1. L. Markus, Structurally stable differential systems, Ann. of Math. (2) 73 (1961), 1-19.

2. M. C. Peixoto and M. M. Peixoto, Structural stability in the plane with enlarged boundary conditions, An. Acad. Brasil. Ci. 31 (1959), 135-160.

3. M. M. Peixoto, On structural stability, Ann. of Math. (2) 69 (1959), 199-222.

4. - Structural stability on two-dimensional manifolds, Topology 1 (1962), 101-120.

Johns Hopkins UnIversity 